\title{
Elaboración de Briquetas a partir de Subproductos de Palma Africana (Elaeis guineensis J) y Arroz (Oryza sativa L)
}

\author{
León, Alain' ${ }^{\text {(iD); Santacruz, Stalin }}{ }^{1, *}$ (iD \\ ${ }^{1}$ Universidad Laica "Eloy Alfaro" de Manabi, Facultad de Ciencias Agropecuaria, Manta, Ecuador
}

\begin{abstract}
Resumen: El aumento global de la población ha traído como consecuencia mayor demanda de recursos, especialmente fuentes de alimento, influyendo directamente en el desarrollo agroindustrial y en la generación cada vez mayor de subproductos. Ecuador es el sexto país a nivel mundial en producción de aceite de palma africana, además, su producción de arroz es aproximadamente de 1195000 ton. Ambas industrias generan 678000 ton de subproductos/año, los cuales se encuentran subutilizados y representan un problema de manejo para las empresas, trayendo como consecuencia un impacto medioambiental. El raquis de palma africana y la cáscara de arroz tienen una composición mayoritariamente de lignina y celulosa confiriéndole un alto potencial para ser usado como combustible en forma de briquetas. En el presente trabajo, la elaboración de briquetas se realizó a partir de mezclas de raquis de palma africana y cáscara de arroz con las siguientes composiciones: $100 \%$ raquis de palma africana; $75 \%$ raquis de palma africana y $25 \%$ cáscara de arroz; $50 \%$ de ambos subproductos, $25 \%$ raquis de palma africana y $75 \%$ de cáscara de arroz; y $100 \%$ cáscara de arroz. Las briquetas fueron analizadas en cuanto a friabilidad y dureza (textura instrumental), para posteriormente utilizar las briquetas con mejores resultados de ese estudio en la determinación de su poder calórico. Se obtuvieron valores de friabilidad entre $0,84 \%$ y $17,86 \%$ y de dureza entre $11,84 \mathrm{~N}$ y $29,44 \mathrm{~N}$, sin que existan diferencias significativas $(\mathrm{p}<0,05)$. El tratamiento escogido con mejores condiciones de friabilidad y dureza (50\% raquis de palma africana y 50\% cáscara de arroz) tuvo un poder calórico de $15,76 \mathrm{MJ} / \mathrm{kg}$.
\end{abstract}

Palabras clave: biomasa; compactación; friabilidad; dureza; poder calórico.

\section{Elaboration of Briquettes from By-products of African Palm (Elaeis guineensis $\mathbf{J}$ ) and Rice (Oryza sativa $\mathrm{L}$ )}

\begin{abstract}
The increase of population all over the world has led to a greater demand for resources, especially food sources, influencing the agro-industrial development and the generation of by-products. Ecuador is the sixth country in the world in palm oil production, besides, its rice production is approximately 1195000 tons. Both industries generate 678000 tons/year of by-products, which are underutilized and represent a disposal problem for companies, resulting in an environmental impact. Palm rachis and rice husk are composed mainly by lignin and cellulose giving it a high potential to be used as a briquette fuel. In the present work, briquettes were made from mixtures of rachis of palm and rice husk with the following compositions: $100 \%$ palm rachis; $75 \%$ palm rachis and $25 \%$ rice husk; $50 \%$ of both by-products; $25 \%$ palm rachis and $75 \%$ rice husk; and $100 \%$ rice husk. The briquettes were analyzed for friability and hardness (instrumental texture). The briquettes with the best characteristics were analyzed for caloric power. The friability varied between $0,84 \%$ and $17,86 \%$ and hardness between $11,84 \mathrm{~N}$ and $29,44 \mathrm{~N}$, without significant differences $(\mathrm{p}<0,05)$. The selected treatment with the best friability and hardness $(50 \%$ palm rachis and $50 \%$ rice husk) had a caloric power of $15,76 \mathrm{MJ} / \mathrm{kg}$.
\end{abstract}

Keywords: biomass; compaction; friability; hardness; caloric power.

\section{INTRODUCCIÓN}

La población a nivel mundial crece de forma acelerada, con una demanda así mismo creciente por recursos limitados, tal es el caso de combustibles fósiles como: petróleo, hulla y sus derivados. Adicionalmente a ello, un aumento considerable de la contaminación ha derivado en una ola de investigaciones destinadas a encontrar nuevas alternativas energéticas, sobre todo aquellas que disminuyan el impacto ambiental (Suárez et al., 2011).

En Estados Unidos y en la Unión Europea, es donde mayor desarrollo se ha alcanzado en el aprovechamiento de subproductos, los cuales han sido utilizados para generar 
energía eléctrica y se han aplicado a materiales de construcción, en estaciones de compostaje y en la generación de energía calorífica principalmente (Saval, 2012). Para el año 2009, el 18,3\% de la energía primaria generada en la Unión Europea fue a partir de fuentes renovables (eólica, hídrica, solar y a partir de biomasa), de la cual casi en su totalidad provino de la biomasa (Berastegui et al., 2017).

El sistema agroindustrial genera gran cantidad de subproductos, los cuales se han incrementado de forma dramática en los últimos años, sobre todo en los países en vías de desarrollo. El impacto medioambiental de dichos subproductos es considerable, ya que en la mayoría de casos las empresas que los generan no tienen el conocimiento o la técnica necesaria para darles un valor agregado, lo que genera un problema de manejo aún sin solución. No obstante, en Latinoamérica se han visto esfuerzos direccionados a aprovechar estos subproductos (Saval, 2012), como materiales para la construcción (Alba et al., 2012) o fuente de energía renovable (Rodríguez y Zambrano, 2010).

La palma africana (Elaeis guineensis $\mathrm{J}$ ) como su nombre lo indica es originaria del continente africano, sin embargo, su cultivo se ha extendido a otras zonas geográficas, como Asia y países del Sur y Centroamérica, particularmente en Ecuador se introdujo en el año de 1953 (Potter, 2011). En los últimos 20 años, en Latinoamérica se ha observado un crecimiento, tanto en la producción de aceite como en áreas de cultivo, liderados por Colombia con un $29 \%$ del total de la superficie sembrada, seguidos de Ecuador con un $24 \%$ y Honduras con un $12 \%$, lo que convierte al Ecuador en el sexto país a nivel mundial en extracción de aceite de palma y el segundo en Latinoamérica región (INIAP, 2018).

La extracción de aceite de palma africana inicia con la separación de las nueces del raquis. Resultado de este proceso se obtiene entre un $70-74 \%$ de residuos sólidos, entre cuesco, fibra y tusas, dando un total anual de 421672 ton. Las tusas son utilizadas en algunas empresas como combustible, debido a su composición que alcanza valores entre 95 y $98 \%$ de celulosa, hemicelulosa y lignina. Para la mayoría de las empresas, estas constituyen un problema de manejo por lo que generalmente desembocan en un problema de contaminación ambiental (Quintero y Torres, 2019).

A la llegada de los colonizadores españoles al nuevo mundo, las culturas nativas basaban su dieta casi en su totalidad en el maíz. Durante los años de conquista el arroz (Oryza sativa L) fue desplazando al maíz poco a poco hasta convertirse en plato principal de los pueblos Latinoamericanos. El Ecuador, para el año 2011 según datos del MAGAP (Ministerio de Agricultura, Ganadería, Acuacultura y Pesca), procesó 1195852 ton de arroz, las cuales produjeron 256429 ton de cáscara. Del total de cáscara solo el $35 \%$ se utiliza en la industria florícola y en criaderos de animales. El material restante se utiliza de la siguiente forma: $50 \%$ es quemado en las piladoras; $15 \%$ es arrojado al borde de las carreteras; $15 \%$ es quemado en sitios aislados; $10 \%$ se quema a la orilla de las carreteras; $5 \%$ es vertida en ríos y el $5 \%$ restante se pierde por la acción de viento (Acero y Rodríguez, 2012).
La cáscara de arroz posee un elevado contenido de sílice, lo que se relaciona directamente con su poco uso en harinas y balanceados para consumo animal. Sin embargo, posee excelentes características para la combustión por su alto contenidos de celulosa $(39,05 \%)$ y lignina $(22,80 \%)$ (Acero y Rodríguez, 2012).

Bolivia, Venezuela y Ecuador son países productores de petróleo, por lo que no deja de ser llamativo que sus poblaciones sean grandes consumidores de leña, misma que se toma directamente de los bosques o selvas, sin que exista información real en cuanto a la cantidad consumida y al impacto que se genera en dichos ecosistemas. Uno de los problemas del uso de leña como combustible es su baja eficiencia calórica (4-6\%), lo que repercute en el uso de grandes cantidades en procesos de combustión. Esto ha resultado en la búsqueda de nuevas alternativas energéticas en aras de reducir el impacto ambiental (De la Torre et al., 2008).

En el Ecuador se han identificado 226 especies diferentes de plantas que son utilizadas como combustible, las cuales se distribuyen de la siguiente forma: 165 se utilizan para fabricación de carbón; 24 como iniciadores de combustión; 29 como sustitutos del petróleo y 8 no han sido especificadas, siendo la Costa la región en la cual más especies se utilizan con 78, seguidas de la Sierra con 59 y la Amazonía con 43 (De la Torre et al., 2008).

Las briquetas, generalmente son productos $100 \%$ ecológicos y lignocelulósicos, clasificados como bioenergía sólida, obtenidos por procesos de densificación o compactación. Estas pueden ser fabricadas mediante bajas o altas presiones. En el primer caso generalmente se utiliza un aglomerante, el cual con frecuencia es de naturaleza biológica. Este tipo de briquetas normalmente presenta un diámetro mayor a $3 \mathrm{~cm}$ (un diámetro inferior es considerado pellet), siendo utilizadas como sustituto de la leña y el carbón vegetal (Martínez, 2015).

Las briquetas a partir de biomasa presentan una ventaja ambiental con respecto al uso de leña, ya que sus emanaciones de $\mathrm{CO}_{2}$ son $50 \%$ menores, además de tener bajas concentraciones de azufre y nitrógeno, gases que influyen directamente sobre el aumento del efecto invernadero y que generan un impacto nocivo sobre el medio ambiente (Berastegui et al., 2017).

Con base en lo descrito anteriormente el objetivo del presente trabajo fue elaborar briquetas a partir de mezclas de subproductos de palma africana y arroz. Las briquetas se analizaron en cuanto a friabilidad y dureza (textura instrumental), y las briquetas con mejores características de friabilidad y dureza se analizaron en cuanto a su poder calorífico.

\section{METODOLOGÍA}

\subsection{Materiales}

El raquis de palma africana se obtuvo en la ciudad de Quinindé, provincia de Esmeraldas, mientras que la cáscara de arroz se consiguió en el cantón 24 de Mayo, provincia de Manabí. 


\subsection{Fabricación de las Briquetas}

Para la fabricación de las briquetas se siguió el método de Berastegui et al. (2017) realizando algunas modificaciones. Con el objetivo de acondicionar el material para el proceso de molienda, tamizado y posterior fabricación de las briquetas, primero se secó la biomasa (solo el raquis de palma africana, ya que el arroz se obtuvo seco). Este secado se realizó con una estufa (Thermo, Electron Corporation, USA) a $70{ }^{\circ} \mathrm{C}$ durante $24 \mathrm{~h}$. Luego del secado, las muestras se redujeron de tamaño en un molino manual (Corona Landersycia, Colombia). Del material molido se tomaron $200 \mathrm{~g}$ de muestra (raquis de palma y cáscara de arroz) y se tamizaron por 2 min con ayuda de una serie de seis tamices (ELE International Forney Inc., USA) con aberturas entre 7E-05 y 9,53E-0,3 m. Posterior al proceso de tamizado se pesaron las fracciones contenidas en cada tamiz y se almacenaron para futuros análisis. Del material tamizado se tomaron las fracciones de mayor cantidad para realizar cinco tratamientos a base de mezclas de raquis de palma africana y cáscara de arroz como se observa en la Tabla 1.

Tabla 1. Composición de las mezclas de subproductos de palma africana y cáscara de arroz, utilizadas para la elaboración de briquetas

\begin{tabular}{cl}
\hline Tratamiento & Composición de la mezcla utilizada $(\%)$ \\
\hline A1 & $100 \%$ raquis de palma africana \\
A2 & $75 \%$ raquis de palma africana $25 \%$ cáscara de arroz \\
A3 & $50 \%$ raquis de palma africana $50 \%$ cáscara de arroz \\
A4 & $25 \%$ raquis de palma africana $75 \%$ cáscara de arroz \\
A5 & $100 \%$ cáscara de arroz \\
\hline
\end{tabular}

Para la preparación del aglutinante se pesó 10,5 g de almidón y se lo mezcló con agua hasta completar $150 \mathrm{~g}$. La mezcla se calentó con agitación constante hasta llegar a una temperatura de $90^{\circ} \mathrm{C}$ con ayuda de una plancha de agitación (Fisher Scientific, USA). Posteriormente, la mezcla se dejó enfriar por 5 min y se le añadió agua para recuperar la pérdida por evaporación durante el calentamiento. Se tomó 40 g del material tamizado (Tabla 1) y se lo mezcló manualmente con la solución aglutinante de almidón previamente preparada. Las mezclas obtenidas se colocaron en moldes de hierro de $0,10 \mathrm{~m}$ de diámetro por $0,25 \mathrm{~m}$ de alto. Sobre los moldes, se colocó un disco de madera de 0,095 m de diámetro por $0,032 \mathrm{~m}$ de alto con el objetivo de facilitar la compactación del material. La compactación se realizó con un proctor (ELE International Forney Inc., USA) de 2,5 kg, que cayó desde una altura de 0,3048 m. Se aplicó una serie de 52 golpes a cada briqueta (Norma ASTM D-1557).

Los moldes con la biomasa compactada se colocaron en un horno (Humboldt, China) a $150{ }^{\circ} \mathrm{C}$ durante $72 \mathrm{~h}$. Transcurrido dicho tiempo se procedió a sacar las briquetas de los moldes. La elaboración de briquetas se realizó por triplicado.

\subsection{Análisis de Friabilidad}

Para el análisis de friabilidad se consideró el estudio de Huanca (2017) con algunas modificaciones. Se determinó el peso por separado de tres briquetas, para luego colocar las mismas en un tambor giratorio artesanal, de $0,15 \mathrm{~m}$ de diámetro por $0,30 \mathrm{~m}$ de largo. El tambor se mantuvo girando durante 1 min a una velocidad aproximada de 132 r.p.m., luego de lo cual se volvió a pesar cada briqueta. La diferencia de peso permitió calcular el porcentaje de pérdida de peso. Los análisis se realizaron por triplicado.

\subsection{Análisis de Dureza (Textura Instrumental)}

Este análisis se basó en el estudio realizado por Huanca (2017) con modificaciones. Se realizó un análisis de penetración utilizando un texturómetro (Shimadzu, Japón) y una aguja de 0,2E-03 $\mathrm{m}$ de diámetro a una velocidad de $3,33 \mathrm{E}-04 \mathrm{~m} / \mathrm{s}$, alcanzando una profundidad de $2 \mathrm{E}-0,2 \mathrm{~m}$. El análisis de penetración se realizó por triplicado a través de la superficie lateral de la briqueta cilíndrica. El resultado reportado fue la resistencia máxima a la penetración, expresada en Newton $(\mathrm{N})$.

\subsection{Cuantificación del poder calórico}

La determinación del poder calorífico bruto se realizó por triplicado, basándose en la Norma ASTM D-240 (Pérez, 2012).

Se determinó el calor de combustión utilizando una bomba calorimétrica o calorímetro. Esta consiste en un recipiente (bomba) donde está contenida la muestra y donde se realizó la combustión. La bomba está sumergida en una cantidad conocida de agua. El sistema completo está contenido en una chaqueta adiabática que impide que el calor salga del sistema. Midiendo las diferencias de temperatura del agua antes y después de la combustión, es posible determinar el calor cedido por el combustible, tomando en cuenta que se tiene un sistema es adiabático, se tiene la siguiente ecuación:

$$
\begin{aligned}
\Delta Q \operatorname{com}=C H 2 O & (T f-T i)+\Delta Q c a l \\
& -\frac{\Delta Q i g n}{m}
\end{aligned}
$$

Donde:

$\Delta Q$ com: calor de combustión

CH2O: calor específico del agua

Tf : Temperatura final del agua

Ti: Temperatura inicial del agua

$\Delta Q$ cal: calor absorbido por el calorímetro

$\Delta Q$ ign: calor de ignición

m: masa de muestra

\subsection{Análisis estadístico}

Para el análisis de resultados, se utilizó el paquete estadístico Infostat (versión 2017). Se hizo uso de un análisis de varianza y una prueba de medias de Tukey con un nivel de significancia del $5 \%$. 


\section{RESULTADOS Y DISCUSIÓN}

\subsection{Análisis granulométrico}

El análisis granulométrico arrojó siete poblaciones para cada uno de los subproductos como se puede ver en las Tablas 2 y 3 .

Se observó que para el caso del raquis de palma africana (Elaeis guineensis $\mathrm{J}$ ) la población más representativa estuvo retenida en el tamiz de abertura de 2E-03 m, con un 26,78\% de la masa total de la muestra (Tabla 2).

Tabla 2. Análisis granulométrico del raquis de palma africana (Elaeis

\begin{tabular}{cc}
\multicolumn{2}{c}{ guineensis J). Fracciones retenidas } \\
\hline $\begin{array}{c}\text { Abertura del tamiz (E- } \\
\mathbf{0 3} \mathbf{~ m})\end{array}$ & $\begin{array}{c}\text { Fracción } \\
\text { porcentual } \\
\text { respecto a la } \\
\text { masa total }(\%)\end{array}$ \\
\hline 9,53 & 22,18 \\
4,75 & 10,34 \\
\hline $\mathbf{2 , 0 0}$ & $\mathbf{2 6 , 7 8}$ \\
\hline 1,80 & 14,55 \\
0,59 & 8,78 \\
0,30 & 10,09 \\
Base & 7,28 \\
\hline
\end{tabular}

Por el contrario, el análisis granulométrico de la cáscara de arroz (Oryza sativa L) mostró que la fracción retenida en el tamiz de abertura 4,1E-04 m tuvo un porcentaje del 61,50\% respecto a la masa total, valor que dobla a la segunda población, misma que fue retenida en el tamiz de abertura $4,75 \mathrm{E}-03 \mathrm{~m}$ con $28,95 \%$ respecto a la masa total.

Tabla 3. Análisis granulométrico de la cáscara de arroz (Oryza sativa L). Fracciones retenidas

\begin{tabular}{cc}
\hline $\begin{array}{c}\text { Abertura del tamiz } \\
(\mathbf{E - 0 3} \mathbf{~ m})\end{array}$ & $\begin{array}{c}\text { Fracción } \\
\text { porcentual } \\
\text { respecto a la masa } \\
\text { total }(\%)\end{array}$ \\
\hline 4,75 & 28,95 \\
\hline $\mathbf{0 , 4 1}$ & $\mathbf{6 1 , 5 0}$ \\
\hline 0,25 & 6,35 \\
0,18 & 1,37 \\
0,15 & 0,81 \\
0,07 & 0,81 \\
Base & 0,21 \\
\hline
\end{tabular}

Las fracciones mayoritarias de raquis de palma africana y cáscara de arroz fueron escogidas para la elaboración de las briquetas.

\subsection{Análisis de Friabilidad}

El análisis de friabilidad mostró que no existió diferencia significativa entre tres tratamientos (A1, A2 y A3), sin embargo, la formulación A4 tuvo la mayor friabilidad y fue diferente significativamente con respecto a las tres anteriores. La quinta formulación (A5) no se analizó debido a que no se logró compactación del material. Aunque no existieron diferencias estadísticas entre los tres primeros tratamientos, se pudo observar un aumento en la friabilidad a medida que los tratamientos disminuían la cantidad presente de raquis de palma africana (Tabla 4).
Tabla 4. Análisis de friabilidad de briquetas a base de mezclas de raquis de palma africana y cáscara de arroz

\begin{tabular}{cc}
\hline Tratamiento & Friabilidad (\% de pérdida de peso) \\
\hline A1 & $0,84^{\mathrm{a}}$ \\
A2 & $3,64^{\mathrm{a}}$ \\
A3 & $5,63^{\mathrm{a}}$ \\
A4 & $17,86^{\mathrm{b}}$ \\
A5 & n.d. \\
\hline
\end{tabular}

Medias con una letra común no son significativamente diferentes $(\mathrm{p}<0,05)$ ${ }^{1}$ : No se pudo elaborar la briqueta por lo que no hay data de friabilidad n.d.: No hay datos

Los tres primeros tratamientos mostraron porcentajes de pérdida de peso menores al $10 \%$, lo que evidencia un producto de buena calidad según la norma española CEN/TS 15210 (Berastegui et al., 2017).

De acuerdo con Berastegui et al. (2017), el tamaño óptimo de partícula para elaborar briquetas oscila entre 1,6E-04 y 6,3E-04 m. Sin embargo, en el presente trabajo solo la fracción escogida de cáscara de arroz estuvo dentro de ese rango de tamaño. A pesar de que las partículas de palma africana tuvieron un mayor tamaño al recomendado, posiblemente la forma de fibras largas de este material pudo contribuir a reducir la friabilidad de las briquetas, dando como resultado valores dentro del rango obtenido para briquetas elaboradas a base de tusa de maíz, carbón mineral y almidón de yuca, con friabilidades entre 2,7 y $96,93 \%$ (Berastegui et al., 2017) y similares a los de briquetas elaboradas con virutas y aserrín de dos tipos de madera, sin utilización de aglomerantes, con friabilidad promedio de 11,32\% (Huanca, 2017).

\subsection{Análisis de Dureza (Textura Instrumental)}

El análisis de dureza mostró que no existieron diferencias significativas entre los cuatro tratamientos, obteniéndose valores medios de resistencia a la penetración que oscilaron entre 11,84 y 29,44 N. El quinto tratamiento no se analizó debido a que no se pudo formar las briquetas. Sin embargo, aunque el análisis estadístico no arrojó diferencias, se observó que a medida que el porcentaje de raquis de palma africana disminuyó, también lo hizo la resistencia a la penetración (Tabla 5). Al parecer la presencia de una mayor cantidad de material fibroso contribuyó a una mejor compactación y dio como resultado un aumento de la dureza de la briqueta.

Valores de dureza entre 228,4 y 253,8 N fueron encontrados par briquetas elaboradas a base de tusa de maíz, carbón mineral y almidón de yuca como aglomerante (Mopoung y Udeye, 2017); y entre 32.2 y $327.3 \mathrm{~N}$ para briquetas de mezclas de fibra de centeno, trigo y colza (Rynkiewicz et al., 2013). La diferencia en resultados puede deberse a los diferentes materiales utilizados, a la diferente densidad de las briquetas o una combinación de ambos.

Tabla 5. Análisis de Dureza (textura instrumental) de briquetas a base de mezclas de raquis de palma africana y cáscara de arroz

\begin{tabular}{cc}
\hline Tratamiento & $\begin{array}{c}\text { Dureza (Resistencia a la penetración en } \\
\text { N) }\end{array}$ \\
\hline A1 & $29,44^{\mathrm{a}}$ \\
A2 & $28,16^{\mathrm{a}}$ \\
A3 & $19,86^{\mathrm{a}}$ \\
A4 & $11,84^{\mathrm{a}}$ \\
A5 & n.d. \\
\hline Medias con una letra común no son significativamente diferentes $(\mathrm{p}<0,05)$ \\
1: No se pudo elaborar la briqueta por lo que no hay data de friabilidad \\
n.d.: No hay datos
\end{tabular}




\subsection{Cuantificación de poder calórico}

Debido a la mayor abundancia de cáscara de arroz y por ende su mayor impacto al medio ambiente, y a que no existió diferencia en friabilidad y dureza entre los tratamientos en estudio, se escogió el tratamiento A3 para las pruebas de cuantificación de poder calorífico. Las briquetas escogidas tuvieron un poder calórico de 15,76 $\mathrm{MJ} / \mathrm{kg}$, valor que se encuentra por encima de briquetas elaboradas a partir de aserrín y virutas cuyo poder calórico fue de 15,17 MJ/kg (Huanca, 2017), tusas de maíz: 15,07 $\mathrm{MJ} / \mathrm{kg}$ (Boarini, 2006), bagazo de caña: 9,21 - 13,82 MJ/kg (Boarini, 2006), pero inferior a las elaboradas con madera: 18,88 MJ/kg (Agostinho-Da Silva et al., 2014) carbón vegetal: 27,63 MJ/kg (Boarini, 2006), mezcla de tallos de rosas y papel: 23,26 MJ/kg (Solórzano, 2017), mezcla de tusas de maíz y carbón mineral: 18,27 MJ/kg (Berastegui et al., 2017) y cáscara de arroz: 16,33 MJ/kg (Boarini, 2006). Cabe señalar que las briquetas fabricadas con tallos de rosas tenían hasta un $30 \%$ de papel en su formulación, material que pudo contribuir al mayor poder calórico. Así mismo las briquetas fabricadas a partir de tusa de maíz y carbón mineral, tuvieron un $40 \%$ de este último material, el cual sin duda contribuyó a incrementar el poder calórico. Si bien las briquetas elaboradas tuvieron un poder calórico menor al de la madera y al del carbón vegetal, es necesario recalcar que las briquetas son elaboradas a partir de material que no es utilizado, provoca contaminación del medio ambiente y produce menores emisiones de elementos contaminantes (azufre) que la quema de leña (Guzmán et al., 2020). La utilización de madera y carbón vegetal provoca afectación a los bosques del Ecuador, por lo que la elaboración de briquetas a partir de subproductos de palma africana y cáscara de arroz abre una posibilidad al uso de madera y carbón vegetal actualmente en uso en Ecuador.

\section{CONCLUSIONES}

Fue posible elaborar briquetas con cuatro de las cinco mezclas estudiadas de raquis de palma africana y cáscara de arroz, siendo la formulación hecha con $100 \%$ de cáscara de arroz la que no permitió formación de briquetas. No se observó diferencias significativas en la dureza entre los cuatro tratamientos evaluados, mientras que respecto a friabilidad solo un tratamiento presentó mayor friabilidad a los otros (25\% raquis de palma africana y $75 \%$ cáscara de arroz). La formulación de la briqueta escogida (50\% raquis de palma africana y $50 \%$ cáscara de arroz) tuvo un poder calórico de 15,76 MJ/kg, menor al de madera y carbón vegetal, pero similar al de las briquetas a base de subproductos del procesamiento de madera, maíz y caña de azúcar.

\section{REFERENCIAS}

Acero, H., \& Rodríguez, J. (2012). Remplazar el uso de diesel por cascarilla de arroz empleado para la generación de vapor. Aspectos técnicos y económicos. [Tesis De grado] Repositorio de la Escuela Superior Politécnica del Litoral (ESPOL), Facultad de Ingeniería en Electricidad y Computación, Guayaquil, Ecuador.

Agostinho-Da Silva, D.; Otomar-Caron, B.; Sanquetta, C.; Behling, A.; Scmidt, D.; Bamberg, R.; Eloy, E. \& DallaCorte, A. (2014). Ecuaciones para estimar el poder calorífico de la madera de cuatro especies de árboles. Revista Chapingo Serie Ciencias Forestales y del Ambiente 20(2), 177-186. https://doi.org/10.5154/r.rchscfa.2013.09.035. Obtenido de: http://www.chapingo.mx/revistas. (Enero, 2020).

Alba, M., Marrero, M., Leiva, C., Montes, M. \& Vilches, L. (2012). Empleo de paneles compuestos por subproductos de centrales térmicas en fachadas trasdosadas. Informes de la Construcción 64(526), 179-190. http://dx.doi.org/10.3989/ic.10.042

ASTM D-1557, J. Bowles (Experimento No. 9), MTC E 115-2000

Berastegui, C., Ortega, J., Mendoza, J., González, Y., \& Gómez, R. (2017). Elaboración de biocombustibles sólidos densificados a partir de tusa de maíz, bioaglomerante de yuca y carbón mineral del departamento de Córdoba. Ingeniare. Revista chilena de ingeniería, 25(4), 643653. , 33052017000400643

Boarini, J. (2006). Utilización del bagazo de caña de azúcar para la elaboración de briquetas de combustibles sólido para usos domésticos en la ciudad de Guatemala. Repositorio Universidad Rafael Landívar, Facultad de Arquitectura y Diseño, Ciudad de Guatemala, Guatemala.

Mopoung, S. \& Udeye, V. (2017). Characterization and Evaluation of Charcoal Briquettes Using Banana Peel and Banana Bunch Waste for Household Heating. American Journal of Engineering and Applied Sciences 10(2), 353-365. http://dx.doi.org/10.3844/ajeassp.2017.353.365

Quintero, L., \& Torres, C. (2019). Análisis de residuos sólidos de palma africana, como alternativa de aprovechamiento de energías renovables en el departamento Cesar. Ingenierías USBMED. 10(1), 8-18. https://doi.org/10.21500/20275846.3662. Medellín, Colombia. Obtenido de: https://revistas.usb.edu.co/index.php/IngUSBmed/articl e/view/3662/3256. (Diciembre, 2019).

Rodríguez, N. \& Zambrano, D. (2010). Los subproductos del café: Fuente de energía renovable. Revista Cenicafé 393, 1-8. Obtenido de: https://biblioteca.cenicafe.org/bitstream/10778/351/1/av t0393.pdf. (Mayo, 2021).

Rynkiewicz, M., Trávníček, P., Krčálová, E. \& Mareček, J. (2013). Influence of annealing temperature of straw briquettes on their density and hardness. Acta Universitatis Agriculturae et Silviculturae Mendelianae Brunensis 61(5), 1377-1382. http://dx.doi.org/ 10.11118/actaun201361051377

Saval, S. (2012). Aprovechamiento de residuos agroindustriales: pasado, presente y futuro. Revista de la Sociedad Mexicana de Biotecnología y Bioingeniería 16(2), 14-46. 
Obtenido

de:

https://www.academia.edu/23775116/Aprovechamiento _de_Residuos_Agroindustriales_Pasado_Presente_y_Fu turo. (Noviembre, 2019)

Solórzano, S. (2017). Elaboración de briquetas a partir de desechos de tallos de rosas y papel reciclado. [Tesis ingeniería]. Repositorio de la Universidad Central del Ecuador, Facultad de Ingeniería Química, Carrera de Ingeniería Química, Quito, Ecuador.

Suárez, J., Martín, G., Sotolongo, J., Rodríguez, E., Savrán, V., Cepero, L., Funes-Monzote, F., Rivero, J., Blanco, D., Machado, R., Martín C., \& García, A. (2011). Experiencias del proyecto BIOMAS-CUBA. Alternativas energéticas a partir de la biomasa en el medio rural cubano. Pasto y Forrajes 34 (4), 473-496. Obtenido de: http://scielo.sld.cu/scielo.php?pid=S086403942011000400007\&script=sci_arttext\&tlng=pt.

(Noviembre, 2019).

\section{BIOGRAFÍAS}

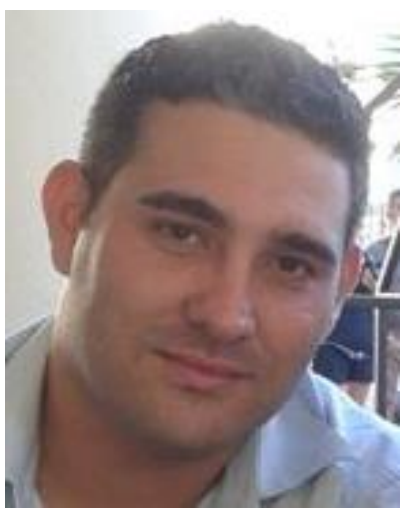

Lázaro Alain León

Álvarez, graduado de Ingeniero Agroindustrial en la Universidad Laica Eloy Alfaro de Manabí y actualmente se desempeña en una empresa purificadora de agua.

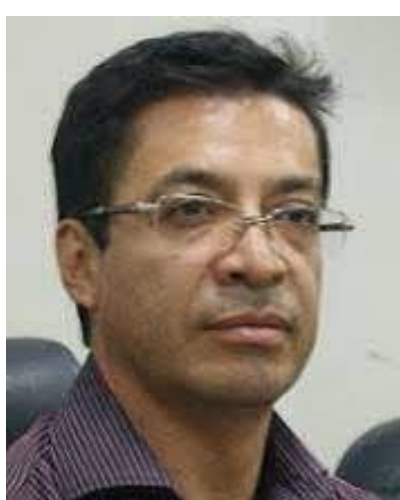

Stalin Santacruz, graduado como Ingeniero Químico en la Escuela Politécnica Nacional, luego de lo cual se desempeñó como asistente de investigación en el entonces Instituto de investigaciones Tecnológicas (actual DECAB). Realizó su maestría en la misma institución, y luego de ello trabajó como investigador en North East Wales Institute (UK). Seguidamente hizo sus estudios de doctorado en Swedish University of Agricultural Sciences, Suecia y en Lund University el postdoctorado. De regreso al Ecuador trabajó como docente en la Universidad San Francisco de Quito y actualmente labora como docente-investigador en la Universidad Laica Eloy Alfaro de Manabí. 